\title{
Improved particle swarm optimization algorithms for economic load dispatch considering electric market
}

\author{
Tan Minh Phan ${ }^{1}$, Phu Trieu Ha ${ }^{2}$, Thanh Long Duong ${ }^{3}$, Thang Trung Nguyen ${ }^{4}$ \\ ${ }^{1}$ Faculty of Electrical and Electronics Engineering, Ton Duc Thang University, Vietnam \\ ${ }^{2}$ Faculty of Electronics-Telecommunications, Saigon University, Vietnam \\ ${ }^{3}$ Faculty of Electrical Engineering Technology, Industrial University of Ho Chi Minh City, Vietnam \\ ${ }^{4}$ Power System Optimization Research Group, Faculty of Electrical and Electronics Engineering, \\ Ton Duc Thang University, Vietnam
}

\section{Article Info}

Article history:

Received Oct 8, 2019

Revised Feb 25, 2020

Accepted Mar 3, 2020

\section{Keywords:}

Competitive electric market

Economic load dispatch

Improved particle Swam

optimization

\begin{abstract}
Economic load dispatch problem under the competitive electric market (ELDCEM) is becoming a hot problem that receives a big interest from researchers. A lot of measures are proposed to deal with the problem. In this paper, three versions of PSO method such as conventional particle swarm optimization (PSO), PSO with inertia weight (IWPSO) and PSO with constriction factor (CFPSO) are applied for handling ELDCEM problem. The core duty of the PSO methods is to determine the most optimal power output of generators to obtain total profit as much as possible for generation companies without violation of constraints. These methods are tested on three and ten-unit systems considering payment model for power delivered and different constraints. Results obtained from the PSO methods are compared with each other to evaluate the effectiveness and robustness. As results, IWPSO method is superior to other methods. Besides, comparing the PSO methods with other reported methods also gives a conclusion that IWPSO method is a very strong tool for solving ELDCEM problem because it can obtain the highest profit, fast converge speed and simulation time.
\end{abstract}

Copyright (C) 2020 Institute of Advanced Engineering and Science. All rights reserved.

\section{Corresponding Author:}

Thang Trung Nguyen,

Power System Optimization Research Group,

Faculty of Electrical and Electronics Engineering,

Ton Duc Thang University,

19 Nguyen Huu Tho street, Tan Phong ward, District 7, Ho Chi Minh City, Vietnam.

Email: nguyentrungthang@tdtu.edu.vn

$\begin{array}{ll}\text { NOMENCLATURE } \\ \alpha_{1, \alpha_{2}} & \text { Acceleration constants } \\ c_{1, c_{2}, c_{3}} & \text { Coefficients in cost function of the } k t h \text { thermal generating unit } \\ F C_{k} & \text { Cost function of the } k t h \text { unit } \\ F_{G b e s t}, F_{B e s t} & \text { The best fitness of the population and each individual } d \\ F P_{D}, F R_{D} & \text { Forecasted demand and forecasted reserve } \\ K_{l}, K_{2}, K_{3} & \text { Penalty factors } \\ L, M a x_{L} & \text { Current iteration and maximum iteration } \\ N G & \text { Number of thermal generating units } \\ P_{k} & \text { Generated power of the } k t h \text { thermal generating unit } \\ r, r_{1}, r_{2} & \text { Randomly generated numbers in the range from } 0 \text { to } 1 \\ R P_{k} & \text { Reserved power of the } k t h \text { thermal generating unit } \\ R P_{k}^{\min }, R P_{k}^{\max } & \text { The minimum and maximum reserved power of the } k t h \text { thermal generating unit } \\ V_{d} & \text { The velocity of the } d t h \text { individual }\end{array}$


$V P_{k}, V R P_{k} \quad$ Velocity of individual corresponding to the updated step size of the generated power and the reserved power of the $k t h$ thermal generating unit

$w_{\min }, w_{\max } \quad$ The minimum and maximum inertia weights

$X_{d}, X_{\text {Best }} \quad$ The position of the $d t h$ individual and the best position of the $d t h$ individual

$X_{\text {Gbest }} \quad$ The best position of all individuals

\section{INTRODUCTION}

To generate electricity for supplying load demand of household and industrial zones, independent thermal power plants spent different expenses. They include operation and management expenses and cost for buying fuels where the fuel cost is considered as the most important part. For this reason, the fuel cost reduction at a possible level is a key mission of the operation process in the power plants. Such work is implemented and presented in economic load dispatch problem (ELD). ELD problem is an optimization strategy in aim to determine the most suitable power output of each thermal generating unit to supply load demand and exactly meet all constraints taken into account. As obtaining the most appropriate power output and satisfying all constraints, total electricity generation cost of all thermal generating units can be possible minimum dependent on the powerful ability of applied optimization tools. Studies on ELD problem have applied different optimization tools and presented in [1-7]. Observing from applications of the methods, it could see that such problem was defined under the centralized electricity market. In this circumstance, companies focused on supplying their generated electricity power with the least total cost. Along with global integration trend, the electrical power companies under the government's management should be changed to private individual one to enhance their competitive ability more effectively [8]. Therefore, ELD problem related to the competitive environment is becoming an urgent issue. Because it boosts innovations in power system operation, scheduling, and control as well as improving service quality and competitiveness of power supplier. In the competitive environment, the core mission of the ELD problem is to determine the most optimal power output of generators to obtain total profit as much as possible for generation companies but without violation of constraints such as active power demand, active power reserve, generation limits and reserve limits [9]. Such problem has been getting the biggest interest of specialists and scientists, and it has been implemented in [10-15] with applications of different algorithms. These algorithms are binary fish swarm method (BFSM) [10], modified teaching learning based optimization technique (MTLBO) [11], binary whale optimization method (BWOM) [12], secant method and invasive weed method (HLR-SM-IWM) [13], memetic binary differential evolution (MBDE) [14], differential evolution (DE) [15], cuckoo search algorithm (CSA) [15] and Hopfield Lagrange network with different functions for determining continuous neuron outputs (HLNEF) [15].

PSO, first formulated by Kennedy and Eberhart in 1995 [16], is a meta-heuristic methodology. The structure of PSO is completely different from genetic algorithm (GA), even though both of the methods are based on the population to find the best solution. In GA, individuals are newly updated according to the principle of natural selection through genetic mechanisms such as mutation, crossover, and reproduction. On the contrary, such individuals in PSO are updated thank to theirs velocity adjustment and the best velocity adjustment. PSO has been considered as one of the algorithms with the simplest calculation model because it has been encoded in a few line commands of computer code with some parameters. Ability and efficiency of PSO have been proven through many optimization problems in power system like reactive power and voltage control considering voltage security assessment [17], optimal design of power system stabilizers [18], solving the short-term hydrothermal coordination [19] and optimal power flow [20].

In this research paper, the conventional PSO and two versions of PSO have been applied to handle ELD problem under the competitive environment to maximize profit of generation plants. These methods have been tested on one three-unit system and one ten-unit system with different constraints. The experimental results obtained from these PSO methods regarding total profit are compared to those from other methods such as DE [9], PSO [9], ALHN [9], PSO [15], CSA [15] and HLN methods [15]. As a result, the contributions of the paper can be given in detail as follows:

- Apply three PSO methods for managing economic load dispatch problem under the competitive electric market.

- Establish an objective function of ELDCEM problem to evaluate all solutions.

- Apply conventional PSO and two improved PSO (IPSO) methods with inertia weight and constriction factor.

- Show strong points of the two IPSO methods over PSO

- Present the whole calculation process of the PSO methods for ELDCEM problem in detail

- Designate the most appropriate control parameters of three PSO methods for each test system. 


\section{PROBLEM FORMULATION}

\subsection{Objective function}

Maximizing total profit is one of the most important tasks in solving the economic load dispatch problem under the competitive electric market [9]. Total profit (TPF) is determined by a difference between total revenue (TR) and total fuel cost (TFC), and formulated as the following model:

$$
T P F=T R-T F C
$$

In (1) the first part on the right side is total revenue (TR). TR is revenue from selling the generated power and reserved power. The price for generated power is called forecasted spot price (FSP) and the price for reserved power is called forecasted reserve price (FRP). Such reserved power is only sold if customers used it. So, the forecasted reserve price is more expensive than the forecasted spot price. It noticed that the reserved power was not used at all time. For evaluating the reserve required and produced, a probability of reserve $(\omega)$ is used [21]. A model for total revenue is calculated by.

$$
T R=F S P \times \sum_{k=1}^{N G} P_{k}+\omega \cdot F R P \cdot \sum_{k=1}^{N G} R P_{k}
$$

The second part is the total fuel cost (TFC). TFC is dependent on number of the power output generated by generators in the plants. In conventional ELD problem, a fuel cost function (FC) for the $k t h$ unit is described as a quadratic function below:

$$
F C_{k}\left(P_{k}\right)=c 1_{k}+c 2_{k} \cdot P_{k}+c 3_{k} \cdot P_{k}^{2} \quad(\$ / h) ; k=1, \ldots, N G
$$

In the competitive electric market, the power reserve demand are always considered. So, power output of each unit in (3) now comprises generated power and reserved power. The new fuel cost function model is formulated by:

$$
F C_{k}\left(P_{k}+R P_{k}\right)=c 1_{k}+c 2_{k} \cdot\left(P_{k}+R P_{k}\right)+c 3_{k} \cdot\left(P_{k}+R P_{k}\right)^{2} \quad(\$ / h) ; k=1, \ldots, N G
$$

The total fuel cost (TFC) model using $\omega$ for determining the reserve required and produced is formed as the following (5):

$$
T F C_{k}=(1-\omega) \cdot \sum_{k=1}^{N G} F C_{k}\left(P_{k}\right)+\omega \cdot \sum_{k=1}^{N G} F C_{k}\left(P_{k}+R P_{k}\right)(\$ / h) ; k=1, \ldots, N G
$$

\subsection{The set of constraints}

The objective function of ELDCEM problem is subjected to some constraints as follows:

- Power demand constraint: The sum of power outputs generated by all units must satisfy the rule as below:

$$
T R=F S P \times \sum_{k=1}^{N G} P_{k}+\omega \cdot F R P . \sum_{k=1}^{N G} R P_{k}
$$

- Power reserve constraint: The total reserved power and reserve demand have a relation as the following (7):

$$
\sum_{k=1}^{N G} R P_{k} \leq F R D
$$

- Generation capacity limit: Active power output of each unit must operate in the range of the minimum power output $\left(P_{k}^{\min }\right)$ and the maximum power output $\left(P_{k}^{\max }\right)$ as follows:

$$
P_{k}^{\min } \leq P_{k} \leq P_{k}^{\max }
$$

- Reserved power limit: Reserved power of each unit is always limited as the following inequality:

$$
R P_{k}^{\min } \leq R P_{k} \leq R P_{k}^{\max }
$$

- Generated and reserved power limit: The sum of the generated power and reserved power of each unit must satisfy the model below:

$$
P_{k}+R P_{k} \leq P_{k}^{\max }
$$




\section{METHOD}

\subsection{Original particle swarm optimization}

Similar to DE, PSO method also had a large variety of applications in engineering fields. Generally, PSO has used a population $(N p)$ to perform the solution update process. Each individual $d$ includes its position $X_{d}$ and velocity $V_{d}$, where $d=1, \ldots, N p$. Such velocity corresponding to each particle $d$ has been updated by its previous velocity, the distance between its current position and its owned best position and the distance between its current position and the best position of its neighbors. New position and velocity of each particle are formulated as the following equations:

$$
\begin{aligned}
& V_{d}^{\text {new }}=V_{d}+\alpha_{1} \cdot r_{1} \cdot\left(X_{\text {Best }}-X_{d}\right)+\alpha_{2} \cdot r_{2} \cdot\left(X_{\text {Gbest }}-X_{d}\right) ; d=1, \ldots, N p \\
& X_{d}^{\text {new }}=X_{d}+V_{d}^{\text {new }} ; d=1, \ldots, N p
\end{aligned}
$$

Although the conventional PSO has been known as a robust and fast tool in solving optimization problems, it still has some downsides in term of easily getting stuck in inefficient search zone and slow convergence for large systems under complex operation conditions and constraints. For that reason, researchers have proposed a different versions to cope with such downsides such as improved particle swarm optimizer (IPSO) [22], inertia weights particle swarm optimization (IWPSO) [23], constriction factor particle swarm optimization (CFPSO) [23] and modified particle swarm optimizer (MPSO) [24]. Such versions of PSO have suggested different improvements to update velocity formula of classical PSO method by using the inertia weights or constriction factor. The improvement detail of two IPSO methods have been presented as.

\subsection{Particle swarm optimization with inertia weight (IWPSO)}

The inertia weight $w$ was the first improved factor. The authors in [22-24] added it into the velocity formula of the original PSO to limit search space of solutions. However, $w$ in $[22,23]$ was a random number and selected by experiences while authors in [24] prop osed a new model for w. This value was changed as the number of iterations altered. The velocity model [24] was presented as follows.

$$
\begin{aligned}
& V_{d}^{\text {new }}=w \cdot V_{d}+\alpha_{1} \cdot r_{1} \cdot\left(X_{\text {Best }}-X_{d}\right)+\alpha_{2} \cdot r_{2} \cdot\left(X_{\text {Gbest }}-X_{d}\right) ; d=1, \ldots, N p \\
& w=w_{\max } \cdot \frac{w_{\max }-w_{\min }}{\operatorname{Max}_{L}} . L ; L=1, \ldots, \operatorname{Max}_{L}
\end{aligned}
$$

\subsection{Particle swarm optimization with constriction factor (CFPSO)}

The constriction factor $(F)$ was a different improvement factor and applied in both [23] and [24]. With the same constriction factor formula, but authors in [24] gave a modification by applying two new acceleration values. The application of the constriction factor was given below [24]:

$$
\begin{aligned}
& V_{d}^{\text {new }}=F \cdot\left[V_{d}+\alpha_{1} \cdot r_{1} \cdot\left(X_{\text {Best }}-X_{d}\right)+\alpha_{2} \cdot r_{2} \cdot\left(X_{\text {Gbest }}-X_{d}\right)\right] ; d=1, \ldots, N p \\
& F=2 /\left|2-\beta-\sqrt{\beta^{2}-4 \beta}\right| ; \beta=\alpha_{1}+\alpha_{2} ; \alpha_{1}=\alpha_{2}=2.05
\end{aligned}
$$

In next section, conventional PSO, IWPSO and CFPSO methods [24] have been used to solve economic load dispatch problem under the competitive electric market.

\section{THE IMPLEMENTATION}

\subsection{Initialization}

In the PSO methods, $N p$ individuals comprises velocity $\left(V_{d}\right)$ and position $\left(X_{d}\right)$. The position of the individual $X_{d}$ includes active generated power $\left(P_{k, d}\right)$ and reserved power $\left(R P_{k, d}\right)$ as shown in (17).

$$
X_{d}=\left\{P_{k, d}, R P_{k, d}\right\} ; d=1, \ldots, N p ; k=1, \ldots, N G
$$

The positions of three PSO methods are initialized as below: 


$$
\begin{aligned}
& P_{k, d}=P_{k}^{\min }+r \cdot\left(P_{k}^{\max }-P_{k}^{\min }\right) \\
& R P_{k, d}=R P_{k}^{\min }+r \cdot\left(R P_{k}^{\max }-R P_{k}^{\min }\right)
\end{aligned}
$$

Similar to position, the velocity of each individual $d$ also includes velocity of active generated power and reserved power and is formed as the following (20).

$$
V_{d}=\left\{V P_{k, d}, V R P_{k, d}\right\} ; d=1, \ldots, N p ; k=1, \ldots, N G
$$

The velocities of three PSO methods are initialized as below: $N p$

$$
\begin{aligned}
& V P_{k, d}=V P_{k}^{\min }+r .\left(V P_{k}^{\max }-V P_{k}^{\min }\right) ; k=1, \ldots, N G ; d=1, \ldots, N p \\
& V R P_{k, d}=V R P_{k}^{\min }+r .\left(V R P_{k}^{\max }-V R P_{k}^{\min }\right) ; k=1, \ldots, N G ; d=1, \ldots, N p
\end{aligned}
$$

In (21) and (22), the minimum and maximum velocities are determined by [25]:

$$
\begin{aligned}
& V P_{k}^{\max }=P_{k}^{\max } ; V P_{k}^{\min }=-V P_{k}^{\max } \\
& V R P_{k}^{\max }=R P_{k}^{\max } ; V R P_{k}^{\min }=-V R P_{k}^{\max }
\end{aligned}
$$

\subsection{Calculate fitness function for each solution}

The fitness function for evaluating all solutions [26] is determined by the following formula:

$$
\text { Fitness }=(T R-T F C)+K_{1} \cdot\left[\sum_{k=1}^{N G}\left(P_{k}\right)-F P_{D}\right]^{2}+K_{2} \cdot\left[\sum_{k=1}^{N G}\left(P R_{k}\right)-F R_{D}\right]^{2}+K_{3} \cdot\left[\sum_{k=1}^{N G}\left(P_{k}+P R_{k}\right)-P_{k}^{\max }\right]^{2}
$$

\subsection{Updating the velocity and position of each individual}

In the PSO algorithms, new solutions are created by updating the velocity and position of each individual [27]. Firstly, such velocity is calculated as presented in section 3 . The velocity value is dependent on four parameters such as the previous velocity, the previous position, the best previous position, and the best global position. Then, such position is computed as (12). For each generation, new solutions are verified for limitations. If a new solution value is lower than the minimum one, it will be set to such minimum value. If the new solution value is higher than the maximum value, it will be set to such maximum value.

\subsection{The whole computing procedure}

The whole search process of the PSO methods for solutions to the considered ELDCEM problem corressponding to the flowchart in Figure 1.

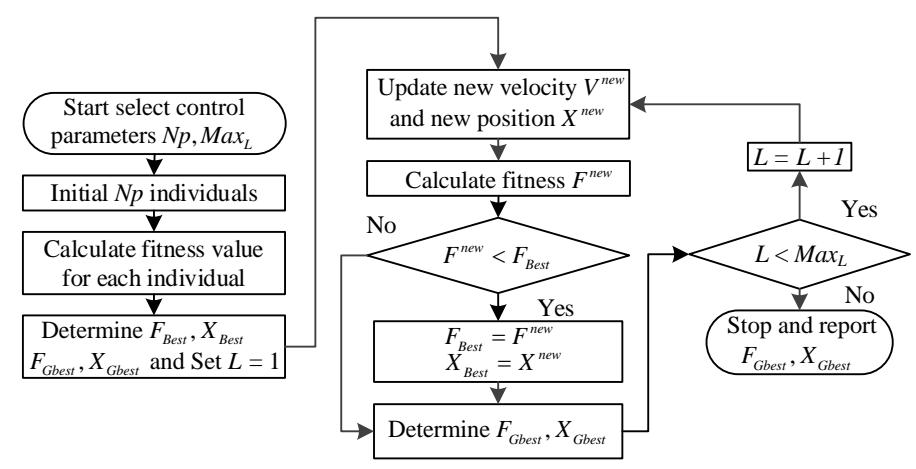

Figure 1. Flowchart for the PSO algorithms 


\section{NUMERICAL RESULTS}

In this portion, the classical PSO, IWPSO, and CFPSO methods have been used to address ELDCEM problem. These methods are tested on three-unit system and ten-unit system with different constraints. The whole data of the two systems are taken from [9]. In the case of three-unit system, 50 successful trials have been implemented and 100 successful trials have been executed for the case of ten-unit system. The work was coded in Matlab program language and run on PC with processor Core i5-2.2 GHz, 4GB of RAM. Additionally, a control parameter selection of three PSO algorithms, such as population size, the maximum number of iterations, inertia weight and constriction factor has described in the next parts because it has played a very important role in finding the best solution. The first two parameters of the PSO algorithms are surveyed for each test system while other ones are determined as in [24].

\subsection{Three-unit system}

\subsubsection{Selecting the most suitable parameters of the PSO methods for the first system}

As mentioned above, a couple of parameters (Np and MaxL) of three PSO methods for the first test system have been pursued to determine the most suitable parameters. For surveying the impact of the parameters, the population is set to 3,5 and 10 , respectively while the maximum iteration is set from 10 to 100. With each value of the maximum iteration, 50 successful trials have been run for PSO, IWPSO and CFPSO methods. The maximum profit obtained by PSO algorithms for the first test system was illustrated in Figures 2, 3 and 4 corresponding to different population, respectively.

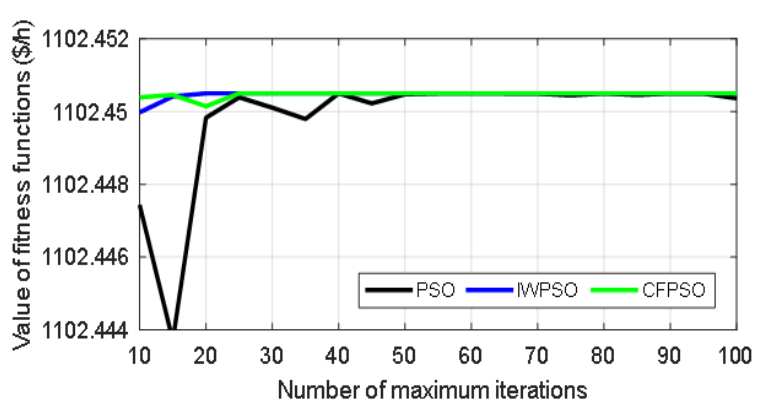

Figure 2. The maximum profit given by 50 trial runs with $N p=3$

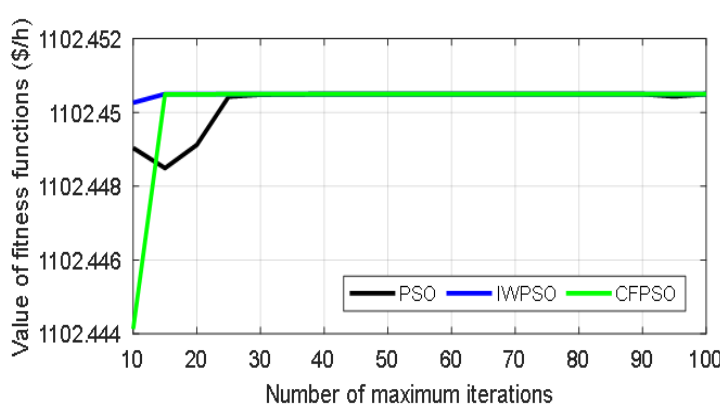

Figure 3. The maximum profit given by 50 trial runs with $N p=5$

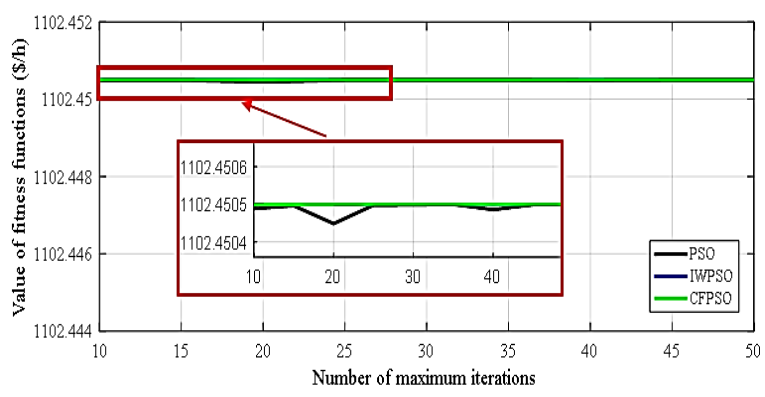

Figure 4. The maximum profit given by 50 trial runs with $N p=10$

The Figures show that the three methods can reach the best profit of $1,102.4502(\$ / \mathrm{h})$ and the profit cannot be higher although the number of iterations continue to be increased. And as the population is increased, the PSO methods can easily find the best solution with smaller number of iterations. Table 1 sees that the highest profit is $1,102.4505(\$ / \mathrm{h})$ and can be reached by setting population to 5 and the maximum iteration to 15. The parameters will be applied for initializing the control parameters of three PSO methods. Besides, the number of evaluations (NFE) is also added in such Table 1. NFE stands for the number of solutions created by the methods and is calculated by the following model [28-29].

$$
N F E=N p \cdot \operatorname{Max}_{L} \cdot N_{c}
$$

In (26), $N_{c}$ is the number of generations in each iteration. For the PSO methods, the number of generations is 1. 
Table 1. Comparisons of optimal parameter results for three cases

\begin{tabular}{lccccc}
\hline & Method & Np & MaxL & NFE & Max. Profit $(\$ / \mathrm{h})$ \\
\hline Survey 1 & PSO & 3 & 50 & 150 & 1102,4437 \\
& CFPSO & 3 & 25 & 75 & 1102,4502 \\
& IWPSO & 3 & 20 & 60 & 1102,4502 \\
Survey 2 & PSO & 5 & 25 & 125 & 1102,4501 \\
& CFPSO & 5 & 15 & 75 & 1102,4504 \\
Survey 3 3 & IWPSO & 5 & 15 & 75 & 1102,4505 \\
& PSO & 10 & 10 & 100 & 1102,4505 \\
& CFPSO & 10 & 10 & 100 & 1102,4505 \\
& IWPSO & 10 & 10 & 100 & 1102,4505 \\
\hline
\end{tabular}

\subsubsection{Result comparisons for the first system}

In this paragraph, three PSO methods are employed for the first system with three units by setting 5 for the population, 15 for the maximum iteration. Table 2 outlines the maximum profit, the mean profit and the minimum profit of all solutions in cooperation with some parameters of PSO, two versions of PSO method and other reported methods. In comparison among the PSO methods, IWPSO and CFPSO methods can get the profits more effectual than PSO method in term of the maximum profit, the mean profit and the minimum profit. It means that they have better capability than PSO in finding the best solution. However, IWPSO is more effective than CFPSO because the IWPSO's maximum profit is $1,102.451(\$ / \mathrm{h})$ whereas that of CFPSO is $1,102.45(\$ / \mathrm{h})$. Besides, PSO has not more stable than two IPSO methods because standard deviation of PSO method is higher than that of other ones. Namely, PSO's standard deviation is 201.9246 while that of IWPSO and CPPSO methods is 166.3297 and 154.7186 , respectively. In addition, results from three implemented PSO methods are also compared to other reported methods, such as DE [9], PSO [9], ALHN [9], DE [15], CSA [15], PSO [15] and HLNEF [15]. From the second column, all methods have the same best profit with $1,102.45(\$ / \mathrm{h})$ except for IWPSO with $1,102.451(\$ / \mathrm{h})$. It can be concluded that these methods can find the best solution to the first test system. However, new solutions produced by the PSO methods are only 75, those of PSO [15], CSA [15] and DE [15] are 2,500, 5,000 and 5,000 respectively while those from DE [9], PSO [9], ALHN [9] and HLNEF [15] are not presented. It can give two comments: Firstly, the simulation time of the PSO methods is faster than other ones. Secondly, the possibility of searching the optimal solutions of IWPSO and CFPSO is more efficient than that of other methods. The solutions obtained by the PSO methods are presented in Table 3.

Table 2. Comparison of result obtained for three-unit system

\begin{tabular}{cccccccc}
\hline Method & Max. Profit $(\$ / \mathrm{h})$ & Mean. Profit $(\$ / \mathrm{h})$ & Min. Profit $(\$ / \mathrm{h})$ & STD & Np & MaxL & NFE \\
\hline DE [9] & $1,102.45$ & 951.37 & 517.94 & 158.23 & - & - & - \\
PSO [9] & $1,102.45$ & 961.39 & 375.00 & 224.50 & - & - & - \\
ALHN [9] & $1,102.45$ & - & - & - & - & - & - \\
DE [9] & $1,102.45$ & 635.3542 & -111.923 & - & 5 & 500 & 5,000 \\
CSA [15] & $1,102.45$ & $1,099.229$ & $1,040.159$ & - & 5 & 500 & 5,000 \\
PSO [15] & $1,102.45$ & 938.8674 & 325 & - & 5 & 500 & 2,500 \\
HLN-EF [15] & $1,102.45$ & 1102.45 & 1102.45 & - & & & - \\
PSO & $1,102.448$ & 999.7856 & 325 & 201.9246 & 5 & 15 & 75 \\
CFPSO & $1,102.450$ & 1023.714 & 492.1755 & 154.7186 & 5 & 15 & 75 \\
IWPSO & $1,102.451$ & 999.8291 & 494.9503 & 166.3297 & 5 & 15 & 75 \\
\hline
\end{tabular}

Table 3. Optimal solution for the three-unit system obtained by the PSO methods

\begin{tabular}{ccccccc}
\hline \multirow{2}{*}{ Unit } & \multicolumn{2}{c}{ PSO } & \multicolumn{2}{c}{ IWPSO } & \multicolumn{2}{c}{ CFPSO } \\
& Pk (MW) & RPk (MW) & Pk (MW) & RPk (MW) & Pk (MW) & RPk (MW) \\
\hline 1 & 325.5026 & 100.0000 & 324.5138 & 100.0000 & 324.4358 & 100.0000 \\
2 & 400.0000 & 0 & 400.0000 & 0 & 400.0000 & 0 \\
3 & 200.0000 & 0 & 200.0000 & 0 & 200.0000 & 0 \\
\hline
\end{tabular}

\subsection{Ten-unit system}

In this case, we've run 100 successful trials for the PSO methods by setting 40 for the population and 60 for the maximum iteration. Results for comparison are summarized in Table 4. The highest profit, the mean profit and the lowest profit are $14,564.74 \$ / \mathrm{h}, 14,479.18 \$ / \mathrm{h}$ and $14,357.24 \$ / \mathrm{h}$, respectively whilst those of PSO method are 14,563.77 \$/h, 14,337.03 $\$ / \mathrm{h}$ and 14,038.64 \$/h, and those of CFPSO method are $14,564.66 \$ / \mathrm{h}, 14,319.77 \$ / \mathrm{h}$ and $14,061.69 \$ / \mathrm{h}$, respectively. In addition, standard deviation of IWPSO 
is two times smaller than that of PSO and CFPSO methods. In comparison to other remaining methods, IWPSO method is ranked the first with the highest profit of 14,564.74 \$/h, HLN-EF [15] is ranked the second position with the second highest profit of 14,564.73 \$/h and DE [15] is put at the last one with the lowest profit of $13,093.1919 \$ / \mathrm{h}$. In consideration of the mean profit and the lowest profit, IWPSO method always obtains better results than other ones excluding HLN-EF [15] and CSA [15]. In consideration of standard deviation value, that of IWPSO is 62.20832 while that of DE [9] and PSO [9] are 9,506 and 11,125, respectively. That of other ones are not found. In relation to converge speed, IWPSO method is faster than these considered methods because it only uses 2,400 evaluations while other considered methods employ from 5,000 to 10,000 evaluations. As a result, IWPSO method is a promising tool for solving this system. The solutions obtained by the PSO methods are presented in Table 5.

Table 4. Comparison of result obtained for ten-unit system

\begin{tabular}{cccccccc}
\hline Method & Max. Profit $(\$ / \mathrm{h})$ & Mean. Profit $(\$ / \mathrm{h})$ & Min. Profit $(\$ / \mathrm{h})$ & STD & Np & MaxL & NFE \\
\hline DE [9] & $14,280.7$ & $2,803.87$ & $2,148.52$ & 9,506 & - & - & - \\
PSO [9] & $14,510.11$ & $2,435.99$ & $4,971.47$ & 11,125 & - & - & - \\
ALHN [9] & $14,564.73$ & - & - & - & - & - & - \\
PSO [15] & $13,158.0653$ & $9,824.8414$ & $6,246.4383$ & - & 10 & 500 & 10,000 \\
CSA [15] & $13,635.105$ & $13,448.0525$ & $13,177.6998$ & - & 10 & 500 & 10,000 \\
DE [15] & $13,093.1919$ & $8,346.2441$ & $3,729.71$ & - & 10 & 500 & 5,000 \\
HLN-EF [15] & $14,564.73$ & $14,564.730$ & $14,564.729$ & - & - & - & - \\
PSO & $14,563.77$ & $14,337.03$ & $14,038.64$ & 149.8791 & 40 & 60 & 2,400 \\
CFPSO & $14,564.66$ & $14,319.77$ & $14,061.69$ & 147.4767 & 40 & 60 & 2,400 \\
IWPSO & $14,564.74$ & $14,479.18$ & $14,357.24$ & 62.20832 & 40 & 60 & 2,400 \\
\hline
\end{tabular}

Table 5. Optimal solution for the ten-unit system obtained by the PSO methods

\begin{tabular}{ccccccc}
\hline Unit & \multicolumn{2}{c}{ PSO } & \multicolumn{2}{c}{ IWPSO } & \multicolumn{2}{c}{ CFPSO } \\
& Pk (MW) & RPk (MW) & Pk (MW) & RPk (MW) & Pk (MW) & RPk (MW) \\
\hline 1 & 455.0000 & 0 & 455.0000 & 0 & 455.0000 & 0 \\
2 & 455.0000 & 0 & 455.0000 & 0 & 455.0000 & 0 \\
3 & 130.0000 & 0 & 130.0000 & 0 & 130.0000 & 0 \\
4 & 130.0000 & 0 & 130.0000 & 0 & 130.0000 & 0 \\
5 & 162.0000 & 0 & 162.0000 & 0 & 162.0000 & 0 \\
6 & 80.0000 & 0 & 80.0000 & 0 & 80.0000 & 0 \\
7 & 25.0000 & 60.0000 & 25.0000 & 60.0000 & 25.0000 & 60.0000 \\
8 & 43.0000 & 0 & 42.9992 & 12.0008 & 42.9872 & 12.0128 \\
9 & 10.0000 & 45.0000 & 10.0000 & 45.0000 & 10.0000 & 45.0000 \\
10 & 10.0000 & 45.0000 & 10.0000 & 32.9991 & 10.0000 & 32.9842 \\
\hline
\end{tabular}

\section{CONCLUSION}

In this paper, the competitive electric market has been considered in classical economic load dispatch problem and the more complicated problem has been solved by three PSO methods. The conventional PSO, IWPSO, and CFPSO methods have been applied to solve two test systems such as three and ten-units with payment model for power delivered. Result comparisons in term of the highest profit, the mean profit and the lowest profit indicate that the optimal solution found by IWPSO method is better than that of CFPSO method while that of PSO is the worst for the two test systems. As a result, IWPSO method was the best method among the PSOs and it can be a representative technique to complete with other ones for such problem. In consideration of the highest profit, IWPSO method and other considered ones can result in the same solution quality for the three-unit system. But for the ten-unit system, the search ability of IWPSO method overtakes that of the others. Furthermore, IWPSO method is the most stable and fastest method because it has a small standard deviation and uses smaller evaluations. For this reason, it can comment that the IWPSO method can be used as a promising optimization technique for ELD problem under the competitive electric market.

\section{ACKNOWLEDGEMENTS}

This research was a part of the scientific research topic with No. CS2019-43. The authors would like to thank Sai Gon University and Ton Duc Thang University, Vietnam. 


\section{REFERENCES}

[1] Dey. T., "Economic load dispatch for multi-generator systems with units having nonlinear and discontinuous cost curves using gravity search algorithm," Int. J. of Applied Power Engineering, vol. 3, no. 3, pp. 166-174, 2014.

[2] Gachhayat. S. K, Dash. S. K, and Ray. P., "Multi objective directed bee colony optimization for economic load dispatch with enhanced power demand and valve point loading," International Journal of Electrical \& Computer Engineering, vol. 7, no. 5. pp. 2382-2391, 2017.

[3] Khamsen. W, Takeang. C, and Aunban. P., "Hybrid method for solving the non smooth cost function economic dispatch problem," International Journal of Electrical and Computer Engineering, vol. 10, pp. 609-616, 2020.

[4] Pham. L. H, et al., "Stochastic fractal search based method for economic load dispatch," TELKOMNIKA Telecommunication Computing Electronics and Control, vol. 17, no. 5, 2019.

[5] Azmi1. A, et al., "The performance of COR optimization using different constraint handling strategies to solve ELD," Indonesian Journal of Electrical Engineering and Computer Science, vol. 17, no. 2, pp. 680-688, 2020.

[6] Van. T. P, Snasel. V, and T. T. Nguyen, "Antlion optimization algorithm for optimal non-smooth economic load dispatch," International Journal of Electrical and Computer Engineering, vol. 10, no. 2, pp. 1187-1199, 2020.

[7] Abdullah. M. N, et al., "Combined economic-emission load dispatch solution using firefly algorithm and fuzzy approach," Indonesian Journal of Electrical Engineering and Computer Science, vol. 16, no. 1, pp. 127-135, 2019.

[8] Orike. S, and Corne. D, "An evolutionary algorithm for bid-based dynamic economic load dispatch in a deregulated electricity market," 13th UK Workshop on Computational Intelligence, pp. 313-320, 2013.

[9] Vo. D. N, Ongsakul. W, and Nguyen. K. P, "Augmented Lagrange Hopfield network for solving economic dispatch problem in competitive environment," AIP Conference Proceedings, vol. 1499, no. 1, pp. 46-53, 2012.

[10] Singhal. P. K, Naresh. R, and Sharma. V," Binary fish swarm algorithm for profit-based unit commitment problem in competitive electricity market with ramp rate constraints," IET Generation, Transmission \& Distribution, vol. 9, no. 13, pp. 1697-1707, 2015.

[11] Krishna. P. R, and Sao. S, "An improved TLBO algorithm to solve profit based unit commitment problem under deregulated environment," Procedia Technology, vol. 25, pp. 652-659, 2016.

[12] Reddy. K. S, et al., "Binary whale optimization algorithm: A new metaheuristic approach for profit-based unit commitment problems in competitive electricity markets," Engineering Optimization, vol. 51, no. 3, pp. 369-389, 2019.

[13] Sudhakar. A. V. V, Karri. C, and Laxmi. A. J, "A hybrid LR-secant method-invasive weed optimisation for profit-based unit commitment," International Journal of Power and Energy Conversion, vol. 9, no. 1, pp. 1-24, 2018.

[14] Dhaliwal. J. S, and Dhillon. J. S, "Profit based unit commitment using memetic binary differential evolution algorithm," Applied Soft Computing, vol. 81, pp. 105502, 2019.

[15] Duong. T. L, et al., "Optimal load dispatch in competitive electricity market by using different models of hopfield lagrange network," Energies, vol. 12, no. 15, pp. 2932, 2019.

[16] Kennady. J, and Eberhart. RC, "Particle swarm optimization," Proc. of IEEE Int. Conf. on Neural Networks, vol. 4, pp. 1942-1948, 1995.

[17] Yoshida. H, et al., "A particle swarm optimization for reactive power and voltage control considering voltage security assessment," IEEE Trans Power Syst, vol. 15, no. 4, pp.1232-1239, 2000.

[18] Abido. M. A., "Optimal design of power system stabilizers using particle swarm optimization," IEEE Trans Energy Conv., vol. 17, no. 3, pp. 406-413, 2002.

[19] Yu. B, Yuan. X, and Wang. J, "Short-term hydro-thermal scheduling using particle swarm optimization method," Energ. Convers. Manag., vol. 48, no. 7, pp. 1902-1908, 2007.

[20] Abido. M. A., "Optimal power flow using particle swarm optimization," Electr Power Energ Syst., vol. 24, no. 7, pp. 563-571, 2002.

[21] Attaviriyanupap. P, et al., "A hybrid LR-EP for solving new profit-based UC problem under competitive environment," IEEE Trans. Power Syst., vol. 18, no. 1, pp. 229-237, 2003.

[22] Shi. Y, and Eberhart. R, "A modified particle swarm optimizer," IEEE international conference on evolutionary computation proceedings. IEEE world congress on computational intelligence, pp. 69-73, 1998.

[23] Eberhart. R. C, and Shi. Y, "Comparing inertia weights and constriction factors in particle swarm optimization," Proceedings of the 2000 congress on evolutionary computation, vol. 1, pp. 84-88, 2000.

[24] Nguyen. T. T, and Vo. D. N, "Improved particle swarm optimization for combined heat and power economic dispatch," Scientia Iranica. Transaction D, Computer Science \& Engineering, Electrical, vol. 23, no. 3, pp. 1318-1334, 2016.

[25] Dubey. B. K, Singh. N. K, and Bhambri. S, "Optimization of PID controller parameters using PSO for two area load frequency control, " IAES International Journal of Robotics and Automation, vol, 8, no.4, pp. 256, 2019.

[26] Abdul-Adheem. W. R, "An enhanced particle swarm optimization algorithm, " International Journal of Electrical and Computer Engineering (IJECE), vol. 9, no. 6, pp. 4904-4907, 2019.

[27] Meziane. M. A, Mouloudi. Y, Draoui. A, "Comparative study of the price penalty factors approaches for Bi-objective dispatch problem via PSO, " International Journal of Electrical and Computer Engineering (IJECE), Vol. 10, No. 4, pp. 3343-3349, 2020.

[28] Nguyen. T. T, "A high performance social spider optimization algorithm for optimal power flow solution with single objective optimization," Energy, vol. 171, pp. 218-240, 2019.

[29] Nguyen. T. T, Quynh. N. V, and Van Dai. L, "Improved firefly algorithm: a novel method for optimal operation of thermal generating units, " Complexity, 2018. 